\title{
Octavio SAlazAR Benítez. La gestación para otros: Una re- flexión jurídico-constitucional sobre el conflicto entre deseos y derechos, Editorial Dykinson, Madrid, 2018, 288 pp., ISBN: 9788491489269.
}

\section{http://dx.doi.org/10.18543/ed-67(1)-2019pp415-422}

El deseo de formar o ampliar una familia empuja, cada vez a más personas, a celebrar contratos cuyo objeto es un recién nacido. Existen agencias especializadas que actúan como intermediadoras entre los padres y las madres intencionales y las mujeres gestantes, y se organizan ferias y foros donde se asesora sobre las mejores opciones para cada familia: hay países donde el contrato tiene un carácter altruista, otros que permiten los contratos con parejas homosexuales, la elección del sexo del bebé, etc. Se ha configurado toda una estructura económica y empresarial pensada para todas aquellas mujeres que «no tienen la capacidad de gestar incluso sometiéndose a técnicas tradicionales de reproducción asistida. $Y$, por supuesto, también para hombres solteros y parejas homosexuales masculinas que quieren formar una familia» ${ }^{1}$. Ante esta realidad, la sociedad en general y los partidos políticos, los medios de

1 Así se pronuncia Surrofair, una de las ferias más importantes sobre gestación subrogada de nuestro país, en su web www.surrofair.com comunicación, las organizaciones civiles, las empresas y la academia en particular, están participando de uno de los debates más polémicos de nuestro tiempo. Al igual que con el aborto o la prostitución, aquí buena parte de la discusión se sitúa en el cuerpo de las mujeres, sobre el que algunos se empeñan en situar la clave del debate.

Disciplinas como la medicina, la tecnología, el derecho, la filosofía, la biología, la economía o la política confluyen, todas ellas, en un tema de absoluta actualidad como es el de los «vientres de alquiler» o la «maternidad subrogada». En este contexto se enmarca el reciente trabajo de Octavio Salazar Benítez, quien se ocupa, desde una perspectiva jurídico-constitucional, de uno de los temas con mayores implicaciones éticas y políticas de nuestro tiempo. Se trata de un trabajo que destaca, por tanto, por su actualidad, por su rigurosidad, por estar profundamente documentado $\mathrm{y}$ por emplear una metodología interdisciplinar a través de la cual se incorporan algunas de las reflexiones que desde otras materias, como la sociología o la filosofía, alimentan el 
debate de la «gestación para otros» (GPO, en adelante), nombre por el que opta el autor. Un trabajo estructurado en ocho capítulos que nos van introduciendo y situando en el estado de la cuestión para, posteriormente, abordar el tema desde una mirada feminista del Derecho constitucional, incluyendo propuestas.

Así, el debate sobre la GPO se empieza a contextualizar desde el primer capítulo, que lleva por nombre «El mercado de los padres felices y las madres ausentes». El autor centra la atención, en primer lugar, en el papel que asumen los medios de comunicación en la construcción de relatos sobre esta práctica. Advierte de que cada vez son más los personajes famosos, nacionales e internacionales, que aparecen en los medios de comunicación dando cuenta de la felicidad de la que gozan tras haber sido padres o madres por medio de la GPO. Por el contrario, es mucho menor el protagonismo que se le da a las mujeres gestantes y, cuando se hace, se realiza desde la exposición de sus experiencias individuales. Parece que esta manera de acceder a la paternidad o a la maternidad alcanza un nivel importante de aceptación social, aunque la información no permite una reflexión mínima sobre las implicaciones éticas y jurídicas que hay detrás. Los partidos políticos también se han sentido interpelados por el debate. Salvo Ciudadanos, que presentó en 2017 una proposición de ley en el Congreso de los Diputados que preveía la gestación por subrogación como un derecho, el resto de fuerzas políticas parecen situarse en contra, aun con fuertes debates internos abiertos.
En el capítulo segundo Octavio Salazar termina de contextualizar la GPO. Bajo el título «La gestación para otros situada» aborda, en primer lugar, la controversia que existe sobre la denominación. Como el autor señala, «las palabras no son inocentes» sino que, por el contrario, resultan relevantes dada su implicación ética y política. Tras analizar nombres como «maternidad subrogada» y «vientres de alquiler», finalmente opta por «gestación para otros», término que, a mi parecer, recoge la esencia del carácter relacional desde el que el autor aborda este debate: quién gesta, para quién se gesta y por qué.

En segundo lugar, sitúa el foco sobre cómo los valores dominantes o hegemónicos del mundo actual impregnan el ámbito de la reproducción y el cuerpo de las mujeres. Por un lado, el derecho está reconociendo la filiación sobre criterios de carácter volitivo o intencional y, por otro lado, algunos de los avances en las técnicas de reproducción asistida están alimentando una noción de mujer y de madre de la que, sin duda, uno de los principales beneficiados es el poder económico. Sin embargo, estas tendencias llevan a algunas autoras a considerar la GPO como una práctica subversiva que permite tanto el cuestionamiento de nociones como la maternidad o la familia basadas en elementos biologicistas, como el acceso de las mujeres al ámbito económico. En esta línea se sitúa, por ejemplo, la jurista Carmel Shalev, con la que el autor dialogará en varias ocasiones a lo largo del trabajo.

Sin embargo, el análisis sobre la GPO no será el adecuado si no 
insertamos esta práctica en el contexto socioeconómico que habitamos y que consta, según Octavio Salazar, de tres pilares fundamentales. El primero versa sobre la ética del modelo neoliberal, que ha construido una sociedad extremadamente individualista, con sujetos cuya identidad se define por lo que consumen y donde los deseos son el motor principal de nuestras acciones. Aquí el cuerpo de las mujeres es un objeto de consumo más sobre el que recae, de manera especial, lo que Luigi Ferrajoli ha denominado «crisis del paradigma constitucional»: que las opciones y aspiraciones personales estén amparadas bajo los derechos fundamentales.

En este marco, y en segundo lugar, la paternidad y la maternidad se fundamentan en aspectos como la intencionalidad y la voluntad. Sin embargo, lo positivo de esta tendencia, apoyada por el avance que el Derecho de Familia ha realizado en el reconocimiento de la diversidad, está en riesgo ante los efectos colaterales y perversos de un doble proceso. Por una parte, se ha avivado el debate sobre la maternidad como elemento esencial de la subjetividad femenina y, aunque la capacidad de elegir tener descendencia es mayor, se ha reforzado también el papel de la mujer en la crianza y en el ámbito doméstico. Por otro lado, se comienza a cuestionar la paternidad tradicional, generando una valoración muy positiva cuando los hombres deciden preocuparse por el cuidado de los hijos e hijas, tarea en la que las mujeres no han contado ni con valoración social ni económica.

El capítulo tercero, que se titula «La autonomía de las mujeres en juego», ofrece una batería de argumentos tanto a favor como en contra de la GPO. Entre los primeros, se reivindica el sueño de formar una familia, aspiración no satisfecha por los procesos de adopción, generalmente más largos y costosos y restringidos en algunos países para familias homosexuales. Además, se considera paternalista la posición de quienes se sitúan en contra de la GPO, pues no reconocen la decisión que la mujer gestante ha adoptado mediante un acuerdo voluntario y libre. Y advierten de que, aunque el primer impulso que lleva a una mujer a querer ser madre gestante sea el económico, con frecuencia esa motivación es desplazada por sentimientos de generosidad y altruismo. Como principales argumentos jurídicos invocan el derecho al libre desarrollo de la personalidad (art. 10.1 CE) y el derecho a crear una familia (art. 39 y varios instrumentos de Derecho Internacional).

En la posición contraria a la GPO convergen razones de carácter religioso y de carácter feminista. Las primeras se apoyan, fundamentalmente, en que esta práctica configura un modelo de familia que se centra en los intereses de los adultos y que cuestiona elementos tan esenciales como el amor materno o la fidelidad conyugal. Desde los posicionamientos feministas, por su parte, se insiste en que la GPO es expresión de la alianza patriarcadocapitalismo, que mercantiliza la capacidad reproductora de las mujeres en favor de una ciudadanía censitaria y que no existen garantías para las mujeres gestantes. Consideran que el deseo de ser padre o madre no supone, en ningún caso, un derecho y en este 
sentido reivindican también instrumentos de Derecho Internacional. Por último, consideran que este tipo de contratos ahondan en la desigualdad de género y merman los derechos de los menores. Para finalizar con el recorrido por los distintos posicionamientos, el autor echa una mirada hacia el exterior y se acerca al estado de la cuestión, desde el plano jurídico y sociopolítico, en Francia, Italia y el Derecho Internacional.

El capítulo cuarto continúa revisando los distintos ordenamientos jurídicos que se pronuncian sobre la GPO. Lleva por título «La gestación para otros desde una perspectiva comparada», y en él se ofrece una panorámica completa de la regulación de esta práctica en los distintos países del mundo. Se centra, especialmente, en la legislación portuguesa, donde se contempla de manera altruista y solo ante casos de imposibilidad de quedar embarazada o de existencia de graves riesgos para la madre o para el hijo derivados del embarazo. Sin embargo, el Tribunal Constitucional luso ha señalado algunos puntos críticos de la norma que versan sobre los discutibles límites de la revocación del consentimiento de la gestante, las dificultades prácticas que puede plantear el control del carácter gratuito del contrato, y el estricto régimen de confidencialidad que, en últimas, excluye el derecho del hijo o hija a conocer su origen. Finaliza con el análisis de las sentencias del TEDH en los casos Menneson y Labassee v. Francia y Paradiso y Campanelli v. Italia. Si bien el Tribunal de Estrasburgo no se pronuncia sobre la adecuación o no de esta práctica al Convenio Europeo de
Derechos Humanos, sí que lo hace sobre el reconocimiento de las relaciones de filiación que se desprenden de la misma, a la luz del art. 8 del Convenio.

El capítulo quinto se adentra en el laberinto jurídico español, tal y como lo califica el autor. Bajo el título «La gestación para otros en el ordenamiento jurídico español: entre el laberinto y el fraude de ley" nos presenta los elementos de la encrucijada. De un lado, el art. 10.1 de la Ley de Técnicas de Reproducción Humana Asistida (LTRHA), en virtud del cual los contratos de GPO son nulos de pleno derecho. Octavio Salazar considera que, además de nulos, estos contratos están prohibidos. Del otro lado, la Instrucción de 5 de octubre de 2010 de la Dirección General del Registro y Notariado, que, bajo la protección del interés del menor y de las mujeres gestantes que renuncian a sus derechos como madres, permite la inscripción de la filiación que se deriva de este tipo de contratos. En otro extremo se encuentra la Ley 20/2011, de 21 de julio, del Registro Civil que, si bien aún no ha entrado en vigor, contempla la posibilidad de que la madre renuncie a la filiación. La luz dentro de este entramado la encontramos, como señala el autor, en el control que desde el Registro se debe hacer sobre la compatibilidad del documento a inscribir con el orden público español. Y ahí el art. 10.1 de la LTRHA actúa, según Octavio Salazar, como límite.

A todo esto se suman también los pronunciamientos sobre la cuestión de los distintos órganos jurisdiccionales y que se analizan en el capítulo. Especialmente, el autor se detiene en el de 
la sala de lo Civil del Tribunal Supremo (de 6 de febrero de 2014, recurso $245 / 2012$ ), en los referidos a las prestaciones por maternidad y paternidad y en la reciente sentencia de la Sala de lo Contencioso del Tribunal Superior de Justicia de Madrid, de 13 de marzo de 2017.

Tras esta presentación del estado de la cuestión profundamente detallada y documentada, llegamos a lo que, a mi parecer, supone la clave del trabajo de Octavio Salazar. El capítulo sexto, titulado «El impacto de género en la gestación para otros», aborda la GPO desde una perspectiva comúnmente ignorada por la doctrina y la jurisprudencia: la de los feminismos jurídicos. Así, el autor atraviesa todo el debate presentado hasta el momento por tres ejes ineludibles: (1) la autonomía reproductiva de las mujeres es un derecho que versa sobre la capacidad de decidir sobre $s u$ propia gestación. Desde esta óptica, la GPO no se enmarca dentro de los derechos de salud reproductiva. (2) Las relaciones de poder que se presentan en el sistema sexo/género condicionan la libertad negocial de las mujeres. Por tanto, y de nuevo en conversación con la jurista Shalev, la noción de dignidad relacional que defiende el autor le hace concluir que el argumento de la libertad no lo puede todo. (3) La autonomía relacional es presupuesto de la dignidad y, desde esta posición, no se sostiene la GPO. Ni siquiera son suficientes los argumentos basados en la lógica del mercado, pues esa misma lógica es la que conduce a las mujeres a situaciones de subordiscriminación, en palabras de Barrère y Morondo $\mathrm{y}$ citadas en el trabajo.
Así, con todas estas claves sobre la mesa, en el capítulo séptimo el autor elabora «Una propuesta teóricamente garantista, y poco probable, de regulación». Una ley que se ocupase de este tema debería garantizar el carácter altruista de la práctica, a pesar de las dudas sobre si efectivamente la GPO puede tener esa naturaleza. El proceso, por su parte, debería ser anónimo o intrafamiliar para evitar los riesgos de explotación y para asegurar, precisamente, el altruismo, y debiera contar con las garantías judiciales oportunas para asegurar los derechos de las partes implicadas. No podría existir discriminación en el acceso, como exigencia del principio de igualdad y no discriminación. Así mismo, deberían establecerse frenos al «turismo gestacional» y controlarse la idoneidad tanto de la mujer gestante como de los padres y/o madres intencionales. Resultaría fundamental para la validez del proceso el consentimiento informado tanto de la mujer gestante como de los padres y madres comitentes. La regulación tendría que ocuparse también de todos los aspectos referidos a la patria potestad. En todo caso, debería garantizar los derechos de la mujer gestante sobre su propio cuerpo, incluido el derecho a poner fin al embarazo, y sus libertades. En relación a los hijos e hijas, estos tendrían reconocido el derecho a conocer su origen. $\mathrm{Y}$ por último, $\mathrm{y}$ para garantizar la eficacia de la norma, tendría que existir un régimen sancionador.

En el último capítulo, Octavio Salazar presenta 10 "Conclusiones desde una ética feminista» sobre la GPO entre las que se encuentran una 
reivindicación de la adopción como forma de acceder a la maternidad o paternidad o la consideración de que la GPO limita la autonomía de las mujeres. En definitiva, conclusiones que reiteran el profundo compromiso del autor con la reflexión en torno a las posibilidades de una sociedad que sitúe como horizonte, según sus propias palabras, la emancipación de las mujeres. Avanzar en esta dirección requiere, sin lugar a dudas, una revisión de los presupuestos del constitucionalismo para permitir una democracia paritaria. Paridad que, como ha defendido el autor en otros trabajos, pasa por la necesaria «revisión de las cláusulas del contrato que partió de una determinada concepción de las subjetividades masculina $y$ femenina $y$ de las relaciones entre ambas» ${ }^{2}$.

Alicia Cárdenas Cordón Universidad de Córdoba

2 SALAZAR BENÍTEZ, Octavio, «Ciudadanía, género y poder: la paridad como principio constitucional», Cuestiones de género: de la igualdad y la diferencia, n. ${ }^{\circ} 10,2015$, p. 30. 
Octavio SAlazAR Benítez. La gestación para otros: Una reflexión jurídico-constitucional sobre el conflicto entre deseos y derechos, Editorial Dykinson, Madrid, 2018, 288 pp., ISBN: 9788491489269.

\author{
http://dx.doi.org/10.18543/ed-67(1)-2019pp415-422
}

\title{
Copyright
}

Estudios de Deusto es una revista de acceso abierto, lo que significa que es de libre acceso en su in tegridad. Se permite su lectura, la búsqueda, descarga, distribución y reutilización legal en cualquier tipo de soporte sólo para fines no comerciales, sin la previa autorización del editor o el autor, siempre que la obra original sea debidamente citada y cualquier cambio en el original esté claramente indicado

Estudios de Deusto is an Open Access journal which means that it is free for full access, reading, search, download, distribution, and lawful reuse in any medium only for non-commercial purposes, without prior permission from the Publisher or the author; provided the original work is properly cited and any changes to the original are clearly indicated. 